\title{
ICTIOSIS FETAL GRAVE
}

\author{
Relato de un caso con sobrevida de más de un año. \\ DrS. HUMBERTO GARCES y FERNANDO DONOSO \\ Cáted ca de Pediatria del Prof. Arturo Seroggie. \\ Hospital "Roberto del Río". Santiago.
}

Hemos estimado de interés publicar esta observación clínica, no sólo por la rareza del cuadro, sino por el hecho, completamente excepcional, de haber logrado sobrevivir más de un año.

La Ictiosis Fetal es una enfermedad congénita que se caracteriza por un engrosamiento generalizado de la capa córnea de la piel, con formación de gruesas láminas epidérmicas, separadas por sur$\cos 0$ fisuras más o menos profundas, en el fondo de las cuales queda el dermis al descubierto. Por este aspecto, la piel ha sido comparada con la de un pez o la de un reptil, de donde los nombres de ictiosis - saurosis, que ha recibido. También se le ha dado otras denominaciones como: Ictiosis intrauterina, Descamación colodionada, Queratosis difusa epidérmica intrauterina, Hiperqueratosis universal congénita, etc., denominaciones todas que hacen mención al aspecto de la piel y a Ja naturaleza congénita de la afección.

Ya hemos dicho que se trata de una anomalía bastante rara, existiendo muy pocos casos de ella descritos en la literatura. En Chile, solamente han sido publicados 4 casos: el primero por Gantes ${ }^{4}$ en 1930 y otros tres de Bustamante, Tejeda y Socías", publicados en la Revista Chilena de Pediatría en 1949. Además sabemos de otros 2 casos de Gaete ${ }^{3}$, que han quedado inéditos.

Etiologia: Es desconocida. Solamente se han formulado algunas hipótesis sobre el particular. Algunos la han atribuído a la Sífilis, sin existir ninguna prueba fehaciente en su apoyo. Para otros, se debe a una carencia en vitamina $A$ en la madre durante el embarazo, basados en el importante papel de esta vitamina en el proceso normal de cornificación de la epidermis. Hay quienes la han atribuido a trastornos endocrinos y desnutrición materna, sin mayores pruebas en su apoyo.

Nosotros nos inclinamos a reconocerla como una enfermedad hereditaria del gru- po de las anomalías constitucionales sistematizadas y nos basamos para pensar así, en que 2 de los casos descritos por Bustamante, Tejeda y Socías ${ }^{2}$ y los 2 de Gaete $^{3}$ eran hermanos, habiendo nacido los primeros en embarazos sucesivos $11^{\circ}$ y $12^{\circ}$ y los dos últimos en los embarazos $2^{\circ}$ y $5^{\circ}$ de un matrimonio en que ambos padres eran primos-hermanos entre sí. Para Arce 1, se trata de una enfermedad de tipo hereditario, que puede tener carácter recesivo $o$ dominante $y$ cita a Siemens, quien tuvo la oportunidad de estudiar una familia a través de 5 generaciones, encontrando 35 sujetos atacados y 32 sanos.

Anatomía Patológica: Sólo existen lesiones de la piel, siendo los restantes organos normales. La alteración reside en la epidermis, cuya capa córnea se encuentra considerablemente engrosada, a causa de un proceso de hiperqueratosis; no hay alteraciones del dermis. Potter ${ }^{5}$ hace referencia a algunos casos en los que se ha encontrado ausencia de las glándulas sudoríparas.

Cuadro Clínico: Se trata casi siempre de niños prematuros, que presentan desde el momento mismo del nacimiento, las lesiones ya descritas de la piel. además de las caracteristicas propias de la prematurez. Toda la superficie cutánea se ve recubierta de gruesas escamas, de color amarillo-grisáceo, separadas por grietas profundas, de intenso color rojo (feto-arlequín). La piel aparece rígida, tensa, brillante, poco flexible y poco elástica, comparable al papel de celofán, debido al intenso proceso de cornificación. La tensión de la piel provoca la formación de ectropion de los párpados y la proyección de los labios hacia adelante, manteniéndose la boca abierta y dejando las encias al descubierto, lo que facilita las infecciones conjuntivales y dificulta mucho la succión, respectivamente.

De acuerdo con la fecha de aparición 
y la intensidad de las lesiones cutáneas, la Ictiosis ha sido clasificada en 3 grados diferentes: una forma grave, que se presenta en niños prematuros, aparece desda el nacimiento y afecta intensamente a toda Ia superficie cutánea; una forma larvada, presente también desde el nacimiento, pero cuyas manifestaciones son menos extensas y se localizan de preferencia alrededor de los orificios naturales (boca, ojos, ano); y una forma tardia, que aparece después del nacimiento, con formación de escamas más finas y que puede ser generalizada o localizada.

Pronóstico: Depende de la forma clínica. En la grave, Ia muerte sobreviene siempre en el curso de las primeras horas o días de la vida, por la misma prematurez o por procesos sépticos adquiridos a través de las grietas cutáneas. En la fortoa larvada, es posible lograr la sobrevida de algunos niños mediante cuidados especiales. La forma tardía tiene mucho mejor pronóstico. Todos los casos publicados en la literatura nacional pertenecen a la forma grave $y$ han fallecido poco después de nacer.

Tratamiento: No existe hasta el momento ninguno efectivo, ni especifico. Se reduce a los cuidados propios de todo ninoo prematuro, a la limpieza de la piel y a la prevención de las infecciones, de puerta de entrada cutánea. También se ha aconsejado la vitamina $A$, que usamos en nuestro paciente durante largo tiempo, sin inconvenientes.

En nuestro caso, hemos empleado la cortisona; por su acción inhibidora de la hiperreactividad celular y limitadora de la formación de tejido colágeno cicatricial, además de su efecto favorable sobre varias dermatopatías de origen desconocido (dermatitis exfoliativa, esclerodermia, dermatomiositis, etc.), nos pareció que podría tener indicación en nuestro enfermo. Los resultados observados no han sido todo lo espectaculares que son, en general, los efectos de la cortisona en muchas afecciones cutáneas, pero tampoco podríamos negar en forma absoluta la posibilidad que esta hormona haya influído en la sobrevida de nuestro paciente.

\section{CASO CLÍNICO}

R. C. A. (Obs. Cl. No 130111).

Antecedentes hereditarios: Padre de 29 años, aparentemente sano, R. Kahn (-). Madre te 25 años, aparentemente sana, R. Kahn (-). No bay consan- guinidad entre ambos padres. Han tenido 4 hijos: 1 de ellos de 2 años, está vivo y es sano, los otros 2 han fallecido a $\operatorname{los} 2$ y 8 meses de edad, respecrivamente, por Trastornos Nutritivos Agudos. El $4^{0}$ hijo es el presente. No acusa aborios. Ninguno de los otros 3 hijos ha presentado anormalidades de la jiel al nater. ni tamporo bay antecedentes de otro caso semejante cn la familia.

Antecedentes oersonates: Nacido en parto ptematuro a $\operatorname{los} 7 \frac{1}{2}$ meses, sin causa aparente. Nació en et domicilio y el parto fué espontáneo. atendido por matrona. Tuvo asfixia leve al nacer. No se pesó.

Enfermedad actual: Es traida al Hospiral "Robetto del Rio", a las pocas horas de nacida, porque le notan Ia piel gruesa en su toralidad y con grietas profundas de color rojo.

Examen "fisico: Recién nacida, de sexo femenino. con peso de $2.080 \mathrm{gr}, \mathrm{y}$ talla de $46 \mathrm{~cm}$. No regula la temperatura (ingresa fria). Al examen general. llama la aterción su aspecto monstruoso. La piel se presenta engrosada, de soperficie lisa. consistencia firme y color amarillo (como de papel celofán), sin petmitir hacet pliegnes. La epidermis se desprende, constítuỵendo láminas córneas de disrinto tamaño, sin producir bemorragia. Entre las láminas se observan grietas. más o menos profundas, de color rojo vivo. Llama la atención la absoluta falta de lanugo y de pelos en las cejas (Fig. 1).

La niña tiene eseasa vitalidad, carece de movimientos espontíneos y toma una posicíón completamente pasiva. La tespitación es regular. saperficial. Hay cierta rigidez de las extremidades. Los reftejos de succión y deglución están presentes, pero el primero de ellos está muy dificultado, por las escamas cótneas y las grietas que rodean la boca.

Cráneo redondeado, pequeño, algo aplanado en h parte posterior. Fontavelas abiettas. pequeñas. deprimidas. Suturas un poco separadas. Ojos: ectropion bila. teral de ambos párpados: cerece de cajas $\mathrm{y}$ de pestañas. Oídos: pabeltón poco desatrolfado. el conducto auditivo está obstraido. Nariz: poco desarrollada. oriftcios nasales semi-obstruidos. Boca: proyección de los labios hacia adelante en forma de "hocico de pescado". labios rigidos con numerosas grietas profundas a nivel de la porsión mucosa. Paladar: ojival. Cuello y columna: nada de especial. Tórax: Corazón: ruidos normales. algo apagados. Pulmones: respiración superifi, cial. sin raidos patológicos. Abdomen: depresible. Cordón ambilical en buenas condiciones. No se palpan visceras. Región ano-genital: edema pubiano. Extremidades: dedos peqneños. con sus extremos aguzados. uñas poco desartolladas.

Didgnóstico de ingreso: Prematurez. lctiosis FetäP.

Evolución: Se le indica alimentación con leche humana, siguiendo el esquema empleado en el Servício de Prematuros. Terramicina (30 mgr. por kg.-peso). Cortisona ( $37 \frac{1}{2}$ mgr. diarios), vitamina A (20.000 U. diarias) y aseo de la piel con vaselina salicilada al $2 \%$. 


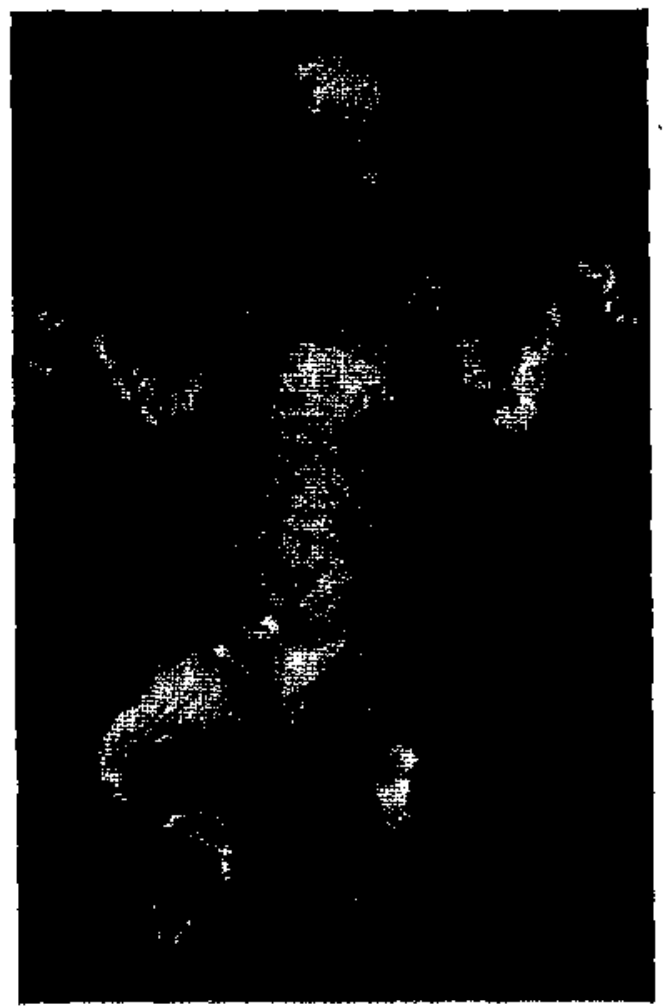

Fighura 1. - Fotografia de la niña recién nacida. Nótese la piel tensa y btillante, como papel de celofán, con numerosas grietas y el desprendimiento de la epidermis en forma de escamas. Eetropion de los párpados y eversión de los labios, que dejan las encías al descu-

bierto. Falta el lanugo y los pelos de las cejas.

La niña permanece en estado grave dumate los 3 primeros dias de su vida. presentando como afección joteccurrence una conjuntivitis supurada (cultivo positivo para el B. de Morax-Axenfeld), que se trató con colicio de penicilina-estreptomisina. Posteriomente comienza a thejorar lentamente sa estado general $y$ a mostrar, poco a poco, mayor vitalidad. La alimentación, que se hacia por medio de una sonda de polietileno. puede realizarse por succión espontánea $y^{a}$ a la semana de edad. aunque con cierta dificultad (por la rigidez de los labios) y con tcndentia a la cianosis (por la obstrueción nasal). La piel se desprende en graesas y duras láminas. como escamas, en toda la superficie del cuerpo. dejando por debajo una aueva capa de piel más fina, de color rojo y de apariencia más normal. El examen hiscológico de las escamas, permitió comprobar que estaban constituidas por la chly córnea de la piel. sin tinguna estructura citológica, aumentada er más de 10 veces de sa grosor normal; Ilama la atención que las laminillas córneas que forman esta capa permanecen uridas consriruyendo un solo block, sin disgregarse en múltiples finas laminillas, como sucede en la piel normal (Fig. 2). Las grietas de la piel, antes descritas, que ban adquirido

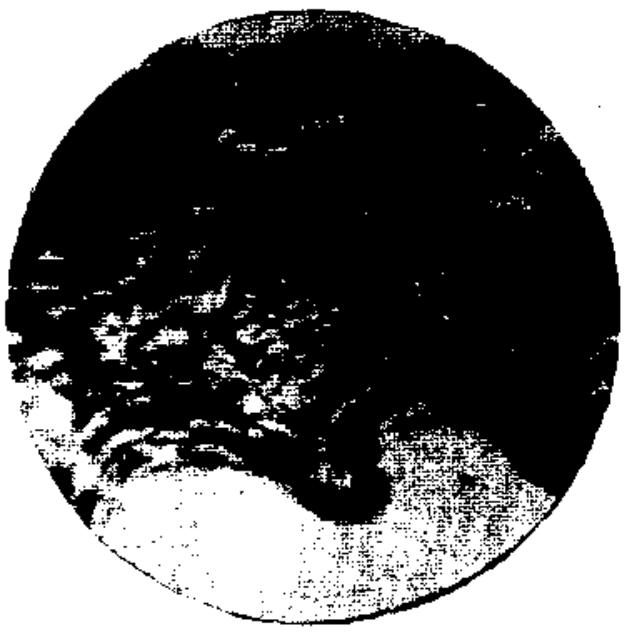

Figura 2. - Corte histológico de una cscama cutánea. Se observa la capa córaea de la epidermis, sin ninguna estructura citóógica, aumentada en $\mathrm{mij}$ de 10 veces del grosor gormal. Llama la atención que las laminillas córneas de esta lapa permanecen unidas constituyendo un solo block, sin disgregarse en muiltiples faminillas. como ocurre en la piel normal.

mayor profundidad a nivel de las superficies de flexión de las grandes articulaciones de los viembros, yan cicatrizando lentamente.

A los 15 días de edad, la niña ya puede llorar $y$ succionar con fuerzos, habiéndose climimado casj totalmente la capa cómea de la piel con que habia nacido, quedando sólo gruesas escamas fímemente adberidas en la frente, regiones periorificiales de la cata y en los pabellones auriculares. Había ido eliminando, una a unu, todas las uñas de las munos y de los pies (Fig. 3).

El tratamiento a base de antibióticos y cortisona se mantuvo por 30 dias, quedando con posterioridad solamente con vitamina $A$ y aseo de la piel con vaselina salicilada. E1 estado general sigue mejorando paulatinamente. El peso. que habia descendido hasta 1.800 gr., permaneció estacionario por 15 dias y lumo la curva de peso tomó un curso ascendente: recuperó el peso de nacimiento a los 32 dias, para luego seguir progresando en forma lenta pero regular. La alimentación con leche humana exclusiva se mantuvo pót 2 meses, complementada con vitaminas $C$ y $D_{i}$ en seguida se hizo cambio paulatino a Eledón, el que se concentró con Nesmida al $1 \%$, para aumentar el aporte de albúmina.

Es interesante señalar que la nucva piel, que quedó después de ser eliminada totalmente la gruesa capa córnea original, tomó nuevamente un aspecro apergaminada y fué, a su tarino, también eliminada. F̀ste fenómeno descrito se repitió varjas veces, pero en cada cambio suresivo, la epidermis iba siendo más arlgada y la piel tomaba un aspecto más cercano del normat. En el carso del tercer mes de su estada presentó una anemia, la que fué corregida con una serie de 3 trans. 


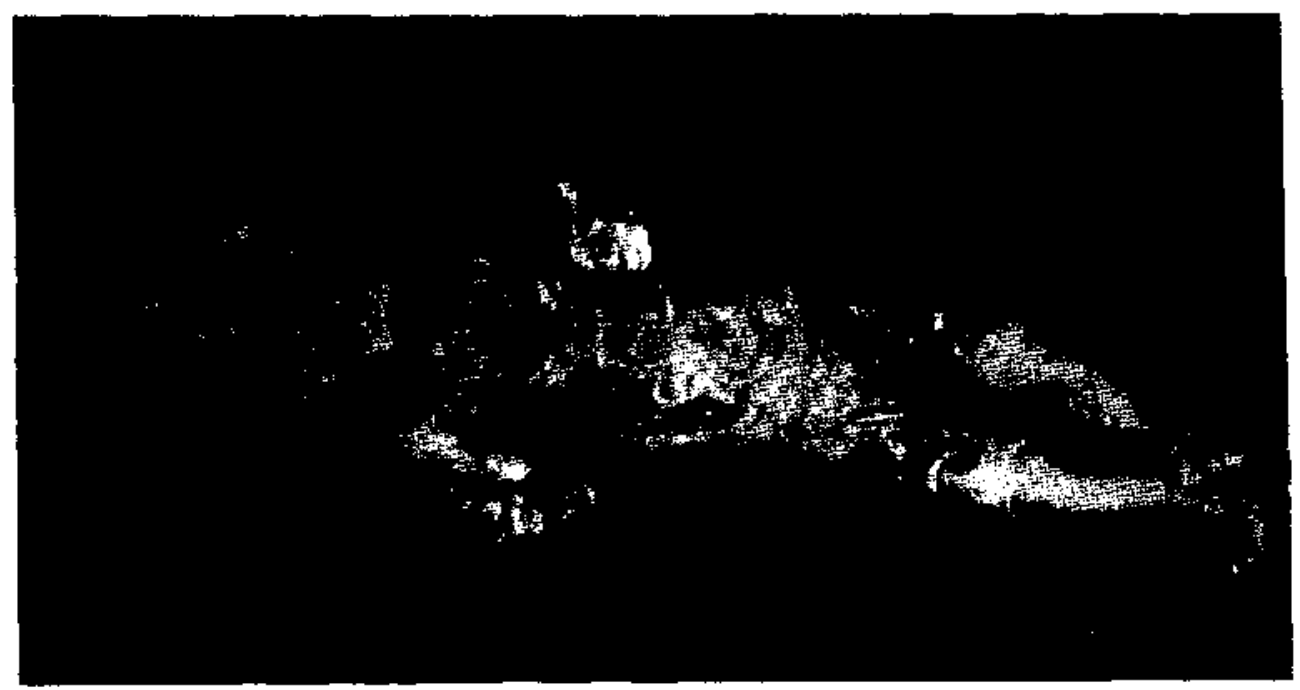

Figura 3. - Aspecto de la niña a los 15 dias de edad. Puede apreciarse un mareado enflaquecimiento, con respecto a la forografía anterior $y$ la eliminación de la epidermis en forma de grandes escamas. constituidas por la capa córnea muy engrosada. Se han caído rodas las uńas.

fasiones de sangre. Permaneció hospitalizada durante 5 meses. siendo dada de alta con un peso de 4.100 gr. y con una alimentación a base de leche de vaca con $3 \%$ de ratcena y $5 \%$ de azúcar. Durante toda su estada hospitalaria recibió vitamina $A$ en dosis de $20.000 \mathrm{U}$. diarias, sint observarse ninguna alteración en los controles radiológicos de los huesos. Dutanze su larga permanencia en el Servicio presentó numerosos cuadros infecciosos intercurrentes, en especial conjuntivitis $y$ bronquitis a repetición, una vez complicada de bronconeumonia, las que cedieron a diversos antibióticos. También contrajo una varicela en el Servicio de Lactantes. a los 3 meses de edad, la que evolucionó en forma atipica por las cacacteristicas especiales de su piel.

La niña ha seguido controlada en el Consultorio N" 12 "San Cristóbal" ₹ en el Hospital "Roberto del Río" por nosotros mismos, habiendo concurtido 2 las edades de 7,10 y 12 meses. Se ha mantenido en bresnas condiciones genetales y se alimenta bien. pero ha subido poco de peso. En el último control. al año de edad. pesaba 5.600 gt. y tenia una talla de $70 \mathrm{~cm}$. a estado nutritivo cra francamente deficiente y la estática bastante atrasada (apenas era capaz de sentarse con apoyo y no se paraba). La piel siempre es anormal. presencando una epidermis seca y engro. sada, que se descama en láminas finas. Persiste ectropion palpebral y cierta rigidez dc las extremidades.

Nota: Fstando redactada esta comrnicatión, nos bemos impuesto del fallecimiento de la riña al año tres meses de edad, a causa de ura bronconeumonia.

\section{RESUMEN}

Se presenta un caso de Ictiosis Fetal Grave en una niña prematura, nacida con un peso de 2.080 gr. Fué tratada con antibióticos, cortisona y vistamina A. Aun cuando este tratamiento no logró producir una mejoria completa del cuadro clínico, por lo menos ha permitido la. sobrevida de la niña por más de un año. Este hecho es digno de destacarse; ya que esta enfermedad produce una mortalidad prácticamente del $100 \%$ en los primeros días de la vida.

\section{SUMMARY}

\section{ICHTHYosis FETALIS gRaVIS.}

A case of Ichthyosis Fetalis Gravis in a premature female, born with a body weight of $2.080 \mathrm{gr}$., is reported. She was treated with antibiotics, cortisone and vitamin A. Even though with this treatment we have not obtained a complete improvement of the clinical picture, at least it has granted the survival of the girl more than one year. This fact must be detached, if we consider that this condition has practically a mortality rate of $100 \%$ in the first few days of life.

\section{BIBLIOGRAFIA}

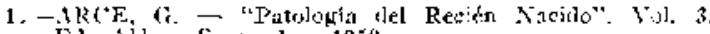
Fil Alilus Santanter. 195il.

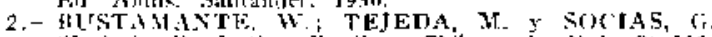
"letosis lieris] Girave". Kev, Chilema de l'ell. 20!111, 1019 .

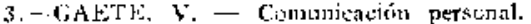

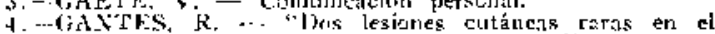
reciću uacido: lularación Cotáuca Durabié del recién natila e Jatiosis fongenitar. Bol. Ie la Clin, Obst. Iruiv, $17: 129,193 \pi$.

5.-POTГER, E. - "j'athology of the Fetus and the New. 19rn". The Year Book Puhl. Inc, Chicago. 1953. 